\title{
Front Matter: Volume 8242
}

, "Front Matter: Volume 8242," Proc. SPIE 8242, Vertical External Cavity Surface Emitting Lasers (VECSELs) II, 824201 (2 March 2012); doi: $10.1117 / 12.923573$

SPIE. Event: SPIE LASE, 2012, San Francisco, California, United States 


\section{PROCEEDINGS OF SPIE}

\section{Vertical External Cavity Surface Emitting Lasers (VECSELS) II}

Anne C. Tropper

Editor

23-24 January 2012

San Francisco, California, United States

Sponsored and Published by

SPIE 
The papers included in this volume were part of the technical conference cited on the cover and title page. Papers were selected and subject to review by the editors and conference program committee. Some conference presentations may not be available for publication. The papers published in these proceedings reflect the work and thoughts of the authors and are published herein as submitted. The publisher is not responsible for the validity of the information or for any outcomes resulting from reliance thereon.

Please use the following format to cite material from this book:

Author(s), "Title of Paper," in Vertical External Cavity Surface Emitting Lasers (VECSELs) II, edited by Anne C. Tropper, Proceedings of SPIE Vol. 8242 (SPIE, Bellingham, WA, 2012) Article CID Number.

ISSN 0277-786X

ISBN 9780819488855

Published by

SPIE

P.O. Box 10, Bellingham, Washington 98227-0010 USA

Telephone +1 3606763290 (Pacific Time) · Fax +1 3606471445

SPIE.org

Copyright () 2012, Society of Photo-Optical Instrumentation Engineers

Copying of material in this book for internal or personal use, or for the internal or personal use of specific clients, beyond the fair use provisions granted by the U.S. Copyright Law is authorized by SPIE subject to payment of copying fees. The Transactional Reporting Service base fee for this volume is $\$ 18.00$ per article (or portion thereof), which should be paid directly to the Copyright Clearance Center (CCC), 222 Rosewood Drive, Danvers, MA 01923. Payment may also be made electronically through CCC Online at copyright.com. Other copying for republication, resale, advertising or promotion, or any form of systematic or multiple reproduction of any material in this book is prohibited except with permission in writing from the publisher. The CCC fee code is 0277-786X/12/\$18.00.

Printed in the United States of America.

Publication of record for individual papers is online in the SPIE Digital Library.

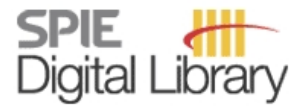

SPIEDigitalLibrary.org

Paper Numbering: Proceedings of SPIE follow an e-First publication model, with papers published first online and then in print and on CD-ROM. Papers are published as they are submitted and meet publication criteria. A unique, consistent, permanent citation identifier (CID) number is assigned to each article at the time of the first publication. Utilization of CIDs allows articles to be fully citable as soon as they are published online, and connects the same identifier to all online, print, and electronic versions of the publication. SPIE uses a six-digit CID article numbering system in which:

- The first four digits correspond to the SPIE volume number.

- The last two digits indicate publication order within the volume using a Base 36 numbering system employing both numerals and letters. These two-number sets start with 00, 01, 02, 03, 04 , $05,06,07,08,09,0 A, 0 B \ldots$. OZ, followed by 10-1Z, 20-2Z, etc.

The CID number appears on each page of the manuscript. The complete citation is used on the first page, and an abbreviated version on subsequent pages. Numbers in the index correspond to the last two digits of the six-digit CID number. 


\section{Contents}

vii Conference Committee

SESSION 1 ULTRAFAST VECSELS I

824203 Picosecond to sub-picosecond pulse generation from mode-locked VECSELs at $1.55 \mu \mathrm{m}$ (Invited Paper) [8242-02]

S. Bouchoule, Z. Zhao, A. Khadour, E. Galopin, J.-C. Harmand, J. Song, G. Aubin, Lab. de Photonique et de Nanostructures, CNRS (France); J. Decobert, Alcatel-Thales III-V Lab. (France); J.-L. Oudar, Lab. de Photonique et de Nanostructures, CNRS (France)

824204 Passively mode-locked GaSb-based VECSELs emitting sub-400-fs pulses at 2 um (Invited Paper) [8242-03]

M. Guina, A. Härkönen, J. Paajaste, J.-P. Alanko, S. Suomalainen, C. Grebing, G. Steinmeyer, Tampere Univ. of Technology (Finland)

824205 Harmonically and fundamentally mode-locked InGaAs-AIGaAs disk laser generating pulse repetition rates in the $100 \mathrm{GHz}$ or pulse durations in the 100-fs range (Invited Paper) [8242-04] U. Griebner, P. Klopp, Max-Born-Institut für Nichtlineare Optik und Kurzzeitspektroskopie (Germany); M. Zorn, M. Weyers, Ferdinand-Braun-Institut (Germany)

\section{SESSION 2 HIGH POWER}

82420620 Watt CW TEMoo intracavity doubled optically pumped semiconductor laser at $532 \mathrm{~nm}$ (Invited Paper) [8242-05]

J. D. Berger, D. W. Anthon, A. Caprara, J. L. Chilla, S. V. Govorkov, A. Y. Lepert, W. Mefferd, Q.-Z. Shu, L. Spinelli, Coherent, Inc. (United States)

824207 High-power quantum dot semiconductor disk lasers (Invited Paper) [8242-06] J. Rautiainen, Tampere Univ. of Technology (Finland); M. Butkus, Univ. of Dundee (United Kingdom); I. Krestnikov, Innolume GmbH (Germany); E. U. Rafailov, Univ. of Dundee (United Kingdom); O. Okhotnikov, Tampere Univ. of Technology (Finland)

824208 Recent advances in the development of yellow-orange GalnNAs-based semiconductor disk lasers (Invited Paper) [8242-07]

T. Leinonen, V.-M. Korpijärvi, A. Härkönen, M. Guina, Tampere Univ. of Technology (Finland)

824209 Strategies for power scaling VECSELs [8242-08]

T.-L. Wang, Y. Kaneda, College of Optical Sciences, The Univ. of Arizona (United States); J. Hader, J. V. Moloney, College of Optical Sciences, The Univ. of Arizona (United States) and Nonlinear Control Strategies Inc. (United States); B. Kunert, W. Stolz, Philipps Univ. Marburg (Germany) and NAsP-III/V GmbH (Germany); S. W. Koch, Philipps-Univ. Marburg (Germany) 
$8242 \mathrm{OB} \quad$ Wavelength tuning of VECSELs by cavity geometry [8242-10]

C. Hessenius, M. Lukowski, J. Moloney, M. Fallahi, College of Optical Sciences, The Univ. of Arizona (United States)

SESSION 4 SINGLE FREQUENCY AND MID-IR

8242 OD GaSb-based semiconductor disk lasers: recent advances in power scaling and narrow linewidth operation (Invited Paper) [8242-12]

J. Wagner, M. Rattunde, T. Töpper, S. Kaspar, B. Rösener, C. Manz, K. Köhler, Fraunhofer-Institut für Angewandte Festkörperphysik (Germany)

8242 OE $\quad$ 589-nm single-frequency VECSEL for sodium guidestar applications [8242-20]

C. Hessenius, P. Y. Guinet, M. Lukowski, J. Moloney, M. Fallahi, College of Optical Sciences, The Univ. of Arizona (United States)

8242 OF Evaluation of the single-frequency operation of a short vertical external-cavity semiconductor laser at $852 \mathrm{~nm}$ [8242-14]

F. A. Camargo, S. Janicot, Lab. Charles Fabry, CNRS, Univ. Paris-Sud (France); I. Sagnes, Lab. de Photonique et de Nanostructures, CNRS (France); A. Garnache, Institut d'Electronique du Sud, CNRS, Univ. Montpellier 2 (France); P. Georges, G. Lucas-Leclin, Lab. Charles Fabry, CNRS, Univ. Paris-Sud (France)

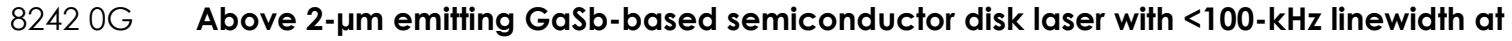
1000-mW output power [8242-15]

S. Kaspar, M. Rattunde, T. Töpper, B. Rösener, C. Manz, K. Köhler, J. Wagner, Fraunhofer-Institut für Angewandte Festkörperphysik (Germany)

$8242 \mathrm{OH} \quad$ IV-VI mid-infrared VECSEL on Si-substrate (Invited Paper) [8242-16]

M. Fill, F. Felder, ETH Zurich (Switzerland) and Phocone AG (Switzerland); M. Rahim, A. Khiar, R. Rodriguez, H. Zogg, ETH Zurich (Switzerland); A. Ishida, Shizuoka Univ. (Japan)

\section{SESSION 5 INTRACAVITY NONLINEAR CONVERSION}

8242 OI VECSELs: non-equilibrium effects and two-color operation (Invited Paper) [8242-17] M. Wichmann, A. Chernikov, M. K. Shakfa, A. Bäumner, M. Koch, Philipps-Univ. Marburg (Germany); M. Scheller, J. Hader, J. V. Moloney, College of Optical Sciences, The Univ. of Arizona (United States); S. W. Koch, Philipps-Univ. Marburg (Germany)

$82420 \mathrm{~J} \quad$ Advances in narrow-linewidth continuous wave semiconductor disk laser pumped optical parametric oscillators (Invited Paper) [8242-18]

N. Hempler, G. Robertson, M Squared Lasers Ltd. (United Kingdom); C. Hamilton, Solus Technologies Ltd. (United Kingdom); G. T. Maker, G. P. A. Malcolm, M Squared Lasers Ltd. (United Kingdom)

$8242 \mathrm{OL} \quad$ 2.7- $\mu \mathrm{m}$ single-frequency TEMoo operation of Sb-based diode-pumped external-cavity VCSEL [8242-13]

A. Laurain, L. Cerutti, M. Myara, A. Garnache, Institut d'Electronique du Sud, CNRS, Univ. Montpellier 2 (France) 
8242 OM UV laser emission around $330 \mathrm{~nm}$ via intracavity frequency doubling of a tunable red AIGalnP-VECSEL [8242-21]

H. Kahle, T. Schwarzbäck, M. Eichfelder, R. Roßbach, M. Jetter, P. Michler, Univ. Stuttgart (Germany)

\section{SESSION $6 \quad$ ULTRAFAST VECSELS II}

$824200 \quad$ Multiphoton imaging with compact semiconductor disk lasers (Invited Paper) [8242-23]

P. Loza-Alvarez, R. Aviles-Espinosa, ICFO - Institut de Ciències Fotòniques (Spain);

D. Artigas-García, ICFO - Institut de Ciències Fotòniques (Spain) and Univ. Politècnica de Catalunya (Spain); C. Hamilton, G. Malcolm, M Squared Lasers LTD (United Kingdom)

8242 OP Frequency-tunable ultrashort pulse VECSEL sources (Invited Paper) [8242-24]

K. G. Wilcox, Univ. of Southampton (United Kingdom)

\section{SESSION 7 ACTIVE REGION DESIGN}

8242 OS Influence of non-radiative carrier losses on pulsed and continuous VECSEL performance (Invited Paper) [8242-27]

A. Laurain, College of Optical Sciences, The Univ. of Arizona (United States); J. Hader, College of Optical Sciences, The Univ. of Arizona (United States) and Nonlinear Control Strategies Inc. (United States); Y.-Y. Lai, T.-L. Wang, College of Optical Sciences, The Univ. of Arizona (United States); M. Yarborough, College of Optical Sciences, The Univ. of Arizona (United States) and Nonlinear Control Strategies Inc. (United States); G. Balakrishnan, T. J. Rotter, P. Ahirwar, The Univ. of New Mexico (United States); J. V. Moloney, College of Optical Sciences, The Univ. of Arizona (United States) and Nonlinear Control Strategies Inc. (United States)

SESSION 8

\section{LATEST CONCEPTS}

8242 OT Non-diffracting beams from surface-emitting lasers (Invited Paper) [8242-28]

G. S. Sokolovskii, M. Butkus, Univ. of Dundee (United Kingdom); S. N. Losev, V. V. Dudelev, A. G. Deryagin, V. I. Kuchinskii, loffe Physico-Technical Institute (Russian Federation); W. Sibbett, Univ. of St. Andrews (United Kingdom); E. U. Rafailov, Univ. of Dundee (United Kingdom)

8242 OW Recent VECSEL developments for sensors applications (Invited Paper) [8242-31]

R. G. Bedford, T. Dang, D. Tomich, Air Force Research Lab. (United States)

\section{POSTER SESSION}

8242 OX Characterization of gain parameters in quantum-dot and quantum-well based VECSEL structures [8242-32]

M. Mangold, V. J. Wittwer, O. D. Sieber, M. Hoffmann, M. Golling, T. Südmeyer, U. Keller, ETH Zurich (Switzerland) 
8242 OY Wetting-layer-pumped continuous wave surface emitting quantum dot laser [8242-33] H. J. Kbashi, A. H. Quarterman, O. J. Morris, Univ. of Southampton (United Kingdom); M. Henini, The Univ. of Nottingham (United Kingdom); A. C. Tropper, K. G. Wilcox, Univ. of Southampton (United Kingdom)

$82420 Z$ Sub-80-fs timing jitter of a stabilized SESAM modelocked VECSEL [8242-34]

V. J. Wittwer, R. van der Linden, ETH Zurich (Switzerland); B. Resan, K. J. Weingarten, Time-Bandwidth Products AG (Switzerland); T. Südmeyer, U. Keller, ETH Zurich (Switzerland)

824210 High-average power femtosecond VECSELs with tunable repetition rates up to $10 \mathrm{GHz}$ [8242-35]

O. D. Sieber, V. J. Wittwer, M. Hoffmann, ETH Zurich (Switzerland); I. L. Krestnikov, Innoume GmbH (Germany); S. S. Mikhrin, D. A. Livshits, Innolume GmbH (Germany); M. Golling, T. Südmeyer, U. Keller, ETH Zurich (Switzerland)

824211 Strain compensation of InGaAs/GaAs SDL gain mirrors grown by molecular beam epitaxy [8242-36]

S. Ranta, T. Leinonen, M. Tavast, T. V. Hakkarainen, I. Suominen, M. Guina, Tampere Univ. of Technology (Finland)

824212 A wavelength tunable 2-ps pulse VECSEL [8242-37]

O. J. Morris, K. G. Wilcox, C. R. Head, A. P. Turnbull, Univ. of Southampton (United Kingdom); P. J. Mosley, Univ. of Bath (United Kingdom); A. H. Quarterman, H. J. Kbashi, Univ. of Southampton (United Kingdom); I. Farrer, H. E. Beere, D. A. Ritchie, Univ. of Cambridge (United Kingdom); A. C. Tropper, Univ. of Southampton (United Kingdom)

$824213 \quad$ MBE growth of electrically pumped VECSELs [8242-38]

M. Golling, W. P. Pallmann, C. A. Zaugg, T. Südmeyer, U. Keller, ETH Zurich (Switzerland)

Author Index 


\title{
Conference Committee
}

\author{
Symposium Chairs
}

Friedhelm Dorsch, TRUMPF Werkzeugmaschinen GmbH + Co. KG (Germany)

Alberto Piqué, Naval Research Laboratory (United States)

Symposium Cochairs

Bo Gu, IPG Photonics Corporation (China)

Andreas Tünnermann, Friedrich-Schiller-Universität Jena (Germany)

Program Track Chair

Klaus P. Streubel, OSRAM GmbH (Germany)

Conference Chair

Anne C. Tropper, University of Southampton (United Kingdom)

Program Committee

Martin D. Dawson, University of Strathclyde (United Kingdom)

Juan L. Chilla, Coherent, Inc. (United States)

Eli Kapon, Ecole Polytechnique Fédérale de Lausanne (Switzerland)

Ursula Keller, ETH Zürich (Switzerland)

Jerome V. Moloney, College of Optical Sciences, The University of Arizona (United States)

Oleg G. Okhotnikov, Tampere University of Technology (Finland)

Edik U. Rafailov, University of Dundee (United Kingdom)

Alexei Sirbu, Ecole Polytechnique Fédérale de Lausanne (Switzerland)

Session Chairs

1 Ultrafast VECSELS I

Ursula Keller, ETH Zürich (Switzerland)

2 High Power

Juan L. Chilla, Coherent, Inc. (United States)

3 Cavity Design

Anne C. Tropper, University of Southampton (United Kingdom) 
$4 \quad$ Single Frequency and Mid-IR

Oleg G. Okhotnikov, Tampere University of Technology (Finland)

5 Intracavity Nonlinear Conversion

Stephane Calvez, University of Strathclyde (United Kingdom)

6 Ultrafast VECSELS II

Uwe Griebner, Max-Born-Institut für Nichtlineare Optik und Kurzzeitspektroskopie (Germany)

$7 \quad$ Active Region Design

Edik U. Rafailov, University of Dundee (United Kingdom)

8 Latest Concepts

Keith Wilcox, University of Southampton (United Kingdom) 